\title{
Determination of the Number of Atoms Trapped in an Optical Cavity
}

\author{
J. McKeever, J. R. Buck, A. D. Boozer, and H. J. Kimble \\ Norman Bridge Laboratory of Physics 12-33, California Institute of Technology, Pasadena, California 91125, USA
}

(Received 16 March 2004; published 27 September 2004)

\begin{abstract}
The number of atoms trapped within the mode of an optical cavity is determined in real time by monitoring the transmission of a weak probe beam. Continuous observation of atom number is accomplished in the strong coupling regime of cavity quantum electrodynamics and functions in concert with a cooling scheme for radial atomic motion. The probe transmission exhibits sudden steps from one plateau to the next in response to the time evolution of the intracavity atom number, from $N \geq 3$ to $N=2 \rightarrow 1 \rightarrow 0$ atoms, with some trapping events lasting over $1 \mathrm{~s}$.
\end{abstract}

Cavity quantum electrodynamics (QED) provides a setting in which atoms interact predominantly with light in a single mode of an electromagnetic resonator [1,2]. Not only can the light from this mode be collected with high efficiency [3], but the associated rate of optical information for determining atomic position can greatly exceed the rate of free-space fluorescent decay employed for conventional imaging [4]. Moreover, the regime of strong coupling, in which coherent atom-cavity interactions dominate dissipation, offers a unique setting for the study of open quantum systems [5]. Dynamical processes enabled by strong coupling in cavity QED provide powerful tools in the emerging field of quantum information science (QIS), including for the realization of quantum computation [6] and distributed quantum networks [7].

With these prospects in mind, experiments in cavity QED have made great strides in trapping single atoms in the regime of strong coupling [4,8-10]. However, many protocols in QIS require multiple atoms to be trapped within the same cavity, with "quantum wiring" between internal states of the various atoms accomplished by way of strong coupling to the cavity field [6,11-13]. Clearly, the experimental ability to determine the number of trapped atoms coupled to a cavity is a critical first step toward the realization of diverse goals in QIS. Experimental efforts to combine ion trap technology with cavity QED are promising [14], but have not yet reached the regime of strong coupling.

In this Letter, we report measurements in which the number of atoms trapped inside an optical cavity is observed in real time. After initial loading of the intracavity dipole trap with $\bar{N} \approx 5$ atoms, the decay of atom number $N \geq 3 \rightarrow 2 \rightarrow 1 \rightarrow 0$ is monitored via the transmission of a near-resonant probe beam, with the transmitted light exhibiting a cascade of "stair steps" as successive atoms leave the trap. After the probabilistic loading stage, the time required for the determination of a particular atom number $N=1,2,3$ is much shorter than the mean interval over which the $N$ atoms are trapped. Hence, this scheme can be used to prepare a precise number of trapped intracavity atoms for subsequent ex- periments in QIS, for which the time scales $\left(g^{-1} \approx\right.$ $\left.\left.10^{-8} \mathrm{~s}\right) \ll(\tau \approx 3 \mathrm{~s})\right)$, where $\tau$ is the atomic trapping time [9] and $\hbar g$ is the atom-field interaction energy. In addition, it requires none of the imaging optics or shielding needed for traditional fluorescence detection from single ions and atoms $[15,16]$, which would be complicated by the presence of our short cavity.

As illustrated in Fig. 1, our experiment combines laser cooling, state-insensitive trapping, and strong coupling in cavity QED [9]. Cs atoms are released from a magnetooptical trap (MOT) several $\mathrm{mm}$ above the cavity, which is formed by the reflective surfaces of mirrors $\left(M_{1}, M_{2}\right)$. Several atoms are cooled and loaded into an intracavity far-off-resonance trap (FORT) and are thereby strongly coupled to a single mode of the cavity. The single-photon Rabi frequency $2 g_{0}$ for one atom at the peak of the Gaussian standing wave is given by $g_{0} / 2 \pi=24 \mathrm{MHz}$, and is based on the reduced dipole moment for the $6 S_{1 / 2}, F=4 \rightarrow 6 P_{3 / 2}, F^{\prime}=4^{\prime}$ transition of the $D 2$ line in Cs at $\lambda_{0}=852.4 \mathrm{~nm}$. Decay rates for the $6 P_{3 / 2}$ excited states and the cavity mode at $\omega_{0}=2 \pi c / \lambda_{0}$ are $\gamma / 2 \pi=$ $2.6 \mathrm{MHz}$ and $\kappa / 2 \pi=4.2 \mathrm{MHz}$, respectively [17]. The fact that $g_{0} \gg(\kappa, \gamma)$ places our system in the strong

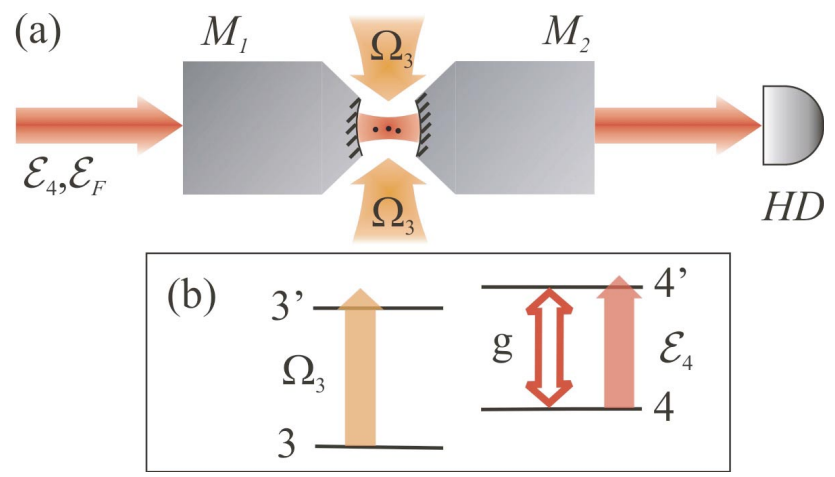

FIG. 1 (color online). Schematic of our experiment. Cs atoms are loaded into an intracavity FORT $\left(\mathcal{E}_{F}\right)$ by way of the transverse cooling field $\Omega_{3}$ and the cavity probe field $\mathcal{E}_{4}$. The transmitted $\mathcal{E}_{4}$ field is directed to a heterodyne detector (HD), allowing real-time determination of intracavity atom number. 
coupling regime of cavity QED, giving critical atom and photon numbers $n_{0} \equiv \gamma^{2} /\left(2 g_{0}^{2}\right) \approx 0.0057, \quad N_{0} \equiv$ $2 \kappa \gamma / g_{0}^{2} \approx 0.037$ [1].

The cavity length $l_{0}=42.2 \mu \mathrm{m}$ is tuned such that it supports $\mathrm{TEM}_{00}$ modes simultaneously resonant with both the $F=4 \rightarrow F^{\prime}=4^{\prime}$ atomic transition at $\lambda_{0}$ and our FORT laser at $\lambda_{F}=935.6 \mathrm{~nm}$. A weak probe laser $\mathcal{E}_{4}$ excites the cavity mode at $\lambda_{0}$ with the cavity output directed to detector HD, while a much stronger trapping laser $\mathcal{E}_{F}$ drives the mode at $\lambda_{F}$. In addition, the region between the cavity mirrors is illuminated by two orthogonal pairs of counter-propagating cooling beams in the transverse plane (denoted $\Omega_{3}$ ), and for $\Omega_{3}=0$, no atoms are detected. Atoms arriving in the region of the cavity mode are exposed to the $\left(\mathcal{E}_{4}, \mathcal{E}_{F}, \Omega_{3}\right)$ fields continuously, with a fraction of the atoms cooled and loaded into the FORT by the combined actions of the $\mathcal{E}_{4}$ and $\Omega_{3}$ fields [9]. For all measurements, the cavity detuning from the $4 \rightarrow 4^{\prime}$ atomic resonance is $\Delta_{C}=0$. The detuning of the $\mathcal{E}_{4}$ probe from the atom-cavity resonance is $\Delta_{4}=$ $+4 \mathrm{MHz}$, and its intensity is set such that the mean intracavity photon number $\bar{n}=0.02$ with no atoms in the cavity. The detuning of the $\Omega_{3}$ transverse cooling field is $\Delta_{3}=+25 \mathrm{MHz}$ from the $F=3 \rightarrow F^{\prime}=3^{\prime}$ resonance, with intensity $I_{3} \approx 4 \times 10^{1} \mathrm{~mW} / \mathrm{cm}^{2}$.

The field $\mathcal{E}_{F}$ that drives the standing-wave, intracavity FORT is linearly polarized, so that all sublevels of the $6 S_{1 / 2}$ ground manifold experience nearly equal ac-Stark shifts [18]. The peak value of the trapping potential is $-U_{0} / h=-47 \mathrm{MHz}$, giving a trap depth $U_{0} / k_{B}=$ $2.2 \mathrm{mK}$ [19]. Importantly, all the $6 P_{3 / 2}$ excited states likewise experience a trapping shift of about $-U_{0}$ (to within $\approx \pm 15 \%)[9,20,21]$, enabling continuous monitoring of trapped atoms in our cavity.

Figure 2(a) displays a typical record of the heterodyne current $i(t)$ resulting from one instance of FORT loading. Here, the current $i(t)$ is referenced to the amplitude of the intracavity field $|\langle\hat{a}\rangle|$ by way of the known propagation and detection efficiencies. The initial sharp drop in $|\langle\hat{a}(t)\rangle|$ shortly after $t=0$ results from atoms that are cooled and loaded into the FORT by the combined action of the $\left(\mathcal{E}_{4}, \Omega_{3}\right)$ fields [9]. Falling atoms are not exposed to $\mathcal{E}_{4}$ until they reach the cavity mode, presumably leading to efficient trap loading for atoms that arrive at a region of overlap between the standing waves at $\left(\lambda_{0}, \lambda_{F}\right)$ for the $\left(\mathcal{E}_{4}, \mathcal{E}_{F}\right)$ fields. The mean atomic free-fall time from our MOT is about $\tau_{F}=0.03 \mathrm{~s}$, and trap loading occurs within a $\pm 10 \mathrm{~ms}$ window around $t=\tau_{F}$ [relative to $t=0$ in Fig. 2(a)], giving a maximum total loading time of about $40 \mathrm{~ms}[8,9]$.

Subsequent to this loading phase, a number of features are apparent in the trace of Fig. 2(a), and are consistently present in all the data. Most notably, the transmission vs time consists of a series of flat "plateaus" in which the field amplitude is stable on long time scales $[15,16]$. Additionally, these plateaus reappear at nearly the same
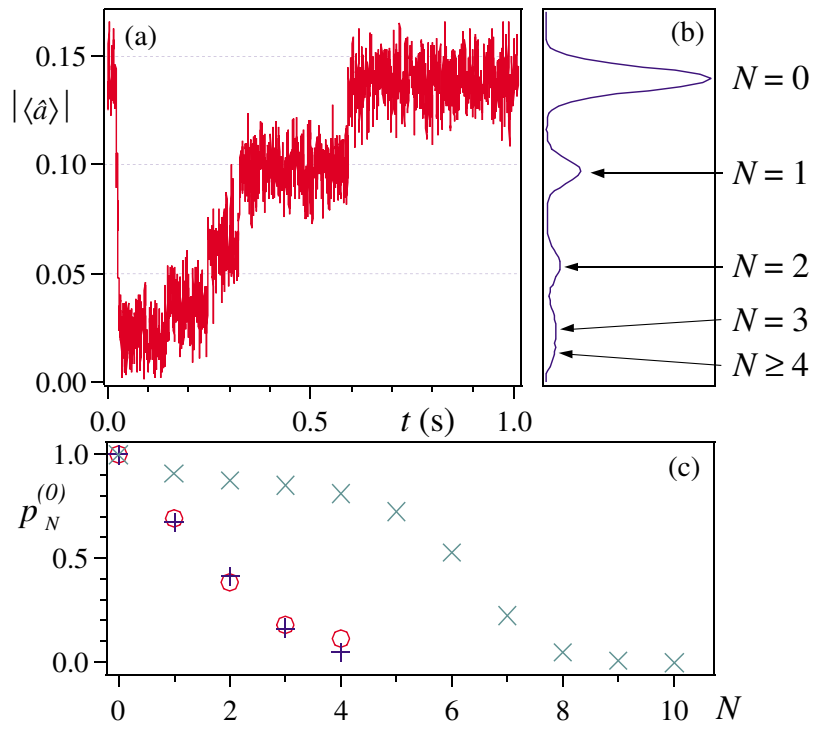

FIG. 2 (color online). (a) A typical detection record in which several $(N>4)$ atoms are loaded into the trap. Heterodyne detection bandwidth $B=1 \mathrm{kHz}$. (b) Histogram of 500 such traces, binned with respect to the heterodyne signal $|\langle\hat{a}\rangle|$. A digital low-pass filter of bandwidth $100 \mathrm{~Hz}$ is applied to each trace prior to the computation of the histogram. (c) Comparison of the model prediction for $p_{0}^{(N)}(y=0.5)(+)$ with the measured positions of the histogram peaks in (b) $(\bigcirc)$. Also shown $(\times)$ is $p_{0}^{(N)}(y=0.1)$ to indicate the possibility to detect specific atom numbers for larger $\bar{N}$.

heights in all repeated trials of the experiment, as evidenced by the histogram of Fig. 2(b). We hypothesize that each of these plateaus represents a different number $N$ of trapped atoms coupled to the cavity mode (arrows in Fig. 2). For detection bandwidth $B, N$ can be measured in time $t_{m} \approx(2 \pi B)^{-1}$, and for our parameters, the uncertainty $p_{\text {err }}$ is dominated by the probability that an atom leaves during the interval $t_{m}$ (for $N=1, p_{\text {err }} \approx 1 \%$ for $B=100 \mathrm{~Hz}$ ).

Consider first the one-atom case, which exhibits relatively large transmission and small variance compared to prior work $[1,4,8,22,23]$. For fixed drive $\mathcal{E}_{4}$, the intracavity field is a function of the coupling parameter $g^{(i, f)}(\mathbf{r})=g_{0} G_{i, f} \sin \left(k_{0} z\right) \exp \left(-2 \rho^{2} / w_{0}^{2}\right)$ where $\rho$ is the transverse distance from the cavity axis $(z), k_{0}=$ $2 \pi / \lambda_{0}$, and $G_{i, f}$ relates to the Clebsch-Gordan coefficient for particular initial and final states $(i, f)$ within the $F=$ $4, F^{\prime}=4^{\prime}$ manifolds. Variations in $g$ as a function of the atom's position $\mathbf{r}$ and internal state might be expected to lead to large variations of the intracavity field, both as a function of time and from atom to atom.

However, one atom in the cavity produces a reasonably well-defined intracavity intensity $I \propto|\langle\hat{a}\rangle|^{2}$ due to the interplay of two effects. The first is that for small probe detunings $\Delta_{4}$, the intracavity intensity $I_{1}$ for one atom is suppressed by a factor $f$ relative to the empty-cavity intensity $I_{0}$, where for weak excitation, $f \approx 4 C_{1}^{2} \gg 1$ 
with $C_{1}=g^{2} / 2 \kappa \gamma$. A persistent, strongly reduced transmission thereby results, since the condition $\left[C_{1}^{(i, f)}(\mathbf{r})\right]^{2} \gg$ 1 is robust to large fluctuations in atomic position $\mathbf{r}$ and internal state. The second effect is that the $F=4 \leftrightarrow F^{\prime}=$ $4^{\prime}$ transition cannot be approximated by a closed, twolevel system, since the $F^{\prime}=4^{\prime}$ excited states decay to both $F=3$, 4 hyperfine ground levels [Fig. 1(b)]. An atom thus spends a fraction $q$ of its time in the cavity QED manifold $\left(4,4^{\prime}\right)$, and a fraction $p \approx 1-q$ in the $\left(3,3^{\prime}\right)$ manifold. In this latter case, the effective coupling is negligible $\left(C_{1}^{e f f} \approx 4 \times 10^{-4}\right)$, leading to an intensity $I \approx$ $I_{0}$. Hence, the intracavity intensity $I(t)$ should approximate a random telegraph signal [15] switching between levels $\left(I_{0}, I_{1}\right)$, with dwell times determined by $\left(\mathcal{E}_{4}, \Omega_{3}\right)$, which in turn set $p$ [24]. Since $\left(\mathcal{E}_{4}, \Omega_{3}\right)$ drive their respective transitions near saturation, the time scale for optical pumping from one manifold to another is $\tau_{P} \sim$ $1 \mu \mathrm{s}$. This time scale represents a fundamental limit to the averaging time for detection, which in our case is $(1 / 2 \pi B) \approx 160 \mu \mathrm{s} \gg \tau_{p}$. The fast modulation of $I(t)$ due to optical pumping processes thereby gives rise to an average detected signal corresponding to intensity $\bar{I}_{1} \approx p I_{0}+q I_{1} \approx p I_{0}$.

This explanation for the $N=1$ case can be extended to $N$ intracavity atoms to provide a simple model for the stair steps of Fig. 2(a). For $N$ atoms, the intracavity intensity should again take the form of a random telegraph signal, now switching between the levels $\left(I_{0}, I_{k}\right)$, with high transmission $I_{0}$ during intervals when all $N$ atoms happen to be pumped into the $\left(3,3^{\prime}\right)$ manifold, and with low transmission $I_{k} \leq I_{1}$ anytime that $1 \leq k \leq N$ atoms reside in the $\left(4,4^{\prime}\right)$ manifold, where $I_{k} \sim I_{1} / k^{2}$ for weak excitation with $\Delta_{C}=\Delta_{4}=0$. The intensities $\left\{I_{k}\right\}$ determine the transition rates $\left\{\gamma_{k \rightarrow k-1}\right\}$ between states with $k$ and $k-1$ atoms in the $\left(4,4^{\prime}\right)$ manifold, while $\Omega_{3}$ determines $\left\{\gamma_{k-1 \rightarrow k}\right\}$ for $k-1 \rightarrow k$ via transitions from the $\left(3,3^{\prime}\right)$ manifold. For the hierarchy of states $k=$ $0,1, \ldots, N$ with transition rates $\left\{\gamma_{k \rightarrow k-1}, \gamma_{k-1 \rightarrow k}\right\}$, the steady-state populations $p_{k}^{(N)}$ can be obtained. With the physically motivated assignments $\gamma_{k-1 \rightarrow k}=\gamma_{0 \rightarrow 1}$ independent of $k$ and $\gamma_{k \rightarrow k-1}=\gamma_{1 \rightarrow 0} / k^{2}$ corresponding to $I_{k} \sim I_{1} / k^{2}$, we find that $p_{0}^{(N)}=1 / \sum_{k=0}^{N}(k !)^{2} y^{k}$, where $y \equiv$ $\gamma_{0 \rightarrow 1} / \gamma_{1 \rightarrow 0}$. Hence, for $I_{k} \ll I_{0}$, the prediction for the average intensity is $\bar{I}_{N} \approx p_{0}^{(N)} I_{0}$, which leads to a sequence of plateaus of increasing heights $\bar{I}_{N+1} \rightarrow \bar{I}_{N} \rightarrow$ $\bar{I}_{N-1}$ as successive atoms leave the trap.

Figure 2(c) compares the prediction of this simple model with the measured values of peak positions in Fig. 2(b). The only adjustable parameter is the value $y=$ 0.5 , giving reasonable correspondence between the model and the measurements. Also shown are values $p_{0}^{(N)}$ for $y=$ 0.1 to indicate that it might be possible to enhance the resolution for a particular range of atom number by framing a given few values $N_{1}, N_{1} \pm 1$ in the transition region $p_{0}^{\left(N_{1}\right)} \approx 0.5$, where $N_{1} \approx 6$ in Fig. $2(\mathrm{c})$. The pa- rameter $y$ would be adjusted via the strengths of the $\left(\mathcal{E}_{4}, \Omega_{3}\right)$ fields.

Although our simple model accounts for the qualitative features in Fig. 2, more detailed correspondence could be obtained using the full master equation for $N$ intracavity atoms, including all Zeeman states and atomic motion [24]. We have made initial progress for the $N=1$ case [25], and are working to extend the treatment to $N \geq 2$.

Figure 3 provides additional evidence for the correspondence between the plateaus in Fig. 2 and atom number. Here, the probe transmission data have been binned not only with respect to the value of $|\langle\hat{a}\rangle|$ as in Fig. 2(b), but also as a function of time. Definite plateaus for $|\langle\hat{a}\rangle|$ are again apparent, but now their characteristic time evolution can be determined. Clearly, the plateaus lying at higher values of $|\langle\hat{a}\rangle|$ correspond to times later in the trapping interval, in agreement with the expectation that $N$ should always decrease with time after the trap-loading window. Moreover, none of the 500 traces in the data set includes a downward step in transmission after the initial trap loading.

Next we consider each atom number individually by integrating the plateau regions along the $|\langle\hat{a}(t)\rangle|$ axis for each time $t$. The dashed horizontal lines in Fig. 3 indicate the boundaries chosen to define the limits of integration for each value of $N$. We thereby obtain time-dependent "populations" $\Phi_{N}(t)$ for $N=0,1,2$, and $\Phi_{\geq 3}(t)=$ $\sum_{N=3}^{\infty} \Phi_{N}(t)$, [see Fig. 4(a)]. The qualitative behavior of these populations is sensible, since almost all trials begin with $N \geq 3$, eventually decaying to $N=2,1,0$.

The quantities $\Phi_{N}(t)$ are approximately proportional to the fraction of experimental trials in which $N$ atoms were trapped at time $t$, so long as the characteristic duration $\Delta t_{N}$ of each plateau far exceeds the time resolution of the detection. If the bandwidth is too low, transient steps no longer represent a negligible fraction of the data, as is the case for transitions between the shortest-lived levels (e.g., $N=3 \rightarrow 2$ ). We estimate that this ambiguity causes uncertainties in $\Phi_{N}$ at the 5\%-10\% level.

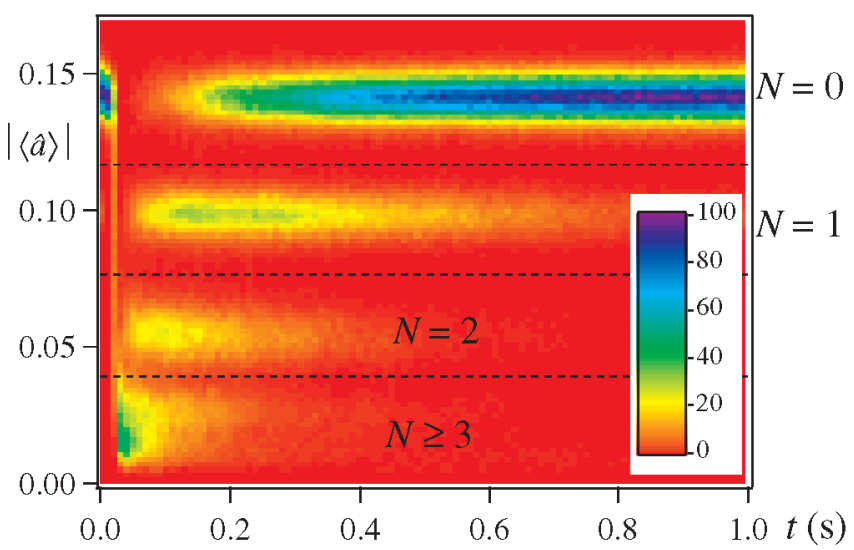

FIG. 3 (color online). Histogram of 500 traces such as the one in Fig. 2(a), binned with respect to both signal strength $|\langle\hat{a}\rangle|$ and time $t$. The signals are filtered first as in Fig. 2(b).

143601-3 


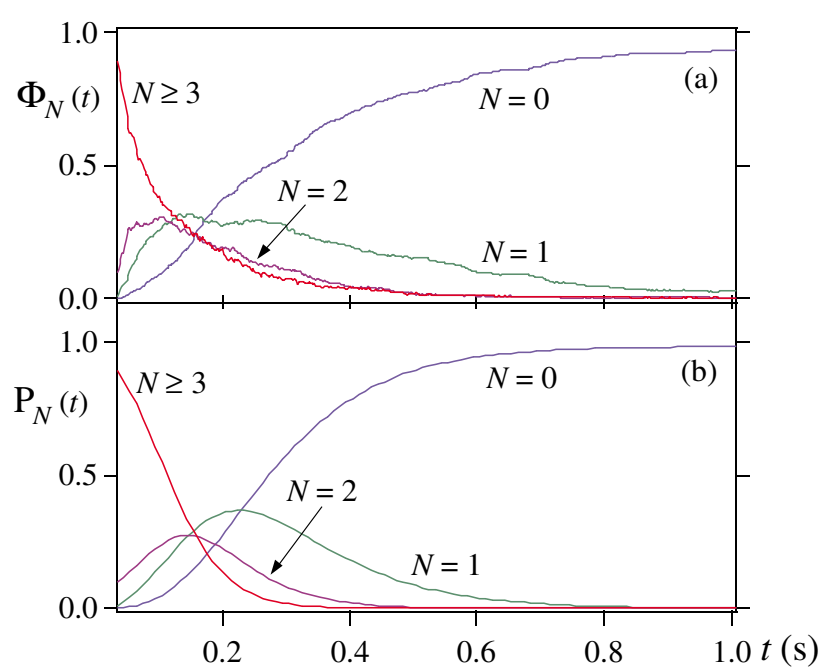

FIG. 4 (color online). (a) Experimentally determined $N$-atom populations $\Phi_{N}(t)$, normalized such that their sum is approximately unity throughout the interval shown. Here, the $t$ axis begins at $t_{0}=0.034 \mathrm{~s}$. (b) The results of a simple model calculation $P_{N}(t)$ are fit to the data $\Phi_{N}(t)$ with one free parameter, the single-atom decay rate $\Gamma$.

Also shown [Fig. 4(b)] is the result of a simple birthdeath model for predicting the time evolution of the populations, namely $\dot{P}_{N}(t)=-\Gamma\left[N P_{N}(t)-(N+\right.$ 1) $\left.P_{N+1}(t)\right]$, where $P_{N}(t)$ represents the probability of $N$ atoms in the trap. We assume that there is one characteristic trap-decay rate $\Gamma$, and that each atom leaves the trap independently of all others. Initial conditions for $N=0$, 1 , and 2 are determined directly from the record of cavity transmission immediately after trap loading, $\Phi_{N}\left(t_{0}\right)$. Since the plateaus for higher values of $N$ are not well resolved, we use a Poisson distribution for $N \geq 3$. The mean $\mu=5.2$ is obtained by solving $\sum_{N=3}^{\infty} e^{-\mu} \mu^{N} / N$ ! = $\Phi_{N \geq 3}\left(t_{0}\right)$. The value $\Gamma=8.5 \mathrm{~s}^{-1}$ used in Fig. $4(\mathrm{~b})$ then results from fitting the analytic solutions $\left\{P_{N}(t)\right\}$ to the data $\left\{\Phi_{N}(t)\right\}$. Although there is reasonable correspondence between Figs. 4(a) and 4(b), $\Phi_{N}(t)$ evolves more rapidly than does $P_{N}(t)$ at early times, and yet the data decay more slowly at long times. This suggests that there might be more than one time scale involved, possibly due to an inhomogeneity of decay rates from atom to atom or to a dependence of the decay rate on $N$. Indeed, we have observed nonexponential decay behavior in other measurements of single-atom trap lifetimes.

Our experiment represents a new method for the realtime determination of the number of atoms trapped and strongly coupled to an optical cavity. We emphasize that an exact number of atoms can be prepared in our cavity within $\approx 200 \mathrm{~ms}$ from the release of the MOT. Although the trap loading is not deterministic, $N$ can be measured quickly compared to the subsequent trapping time $\tau \approx$ $3 \mathrm{~s}$ [9]. These new techniques could assist in the realization of various protocols in quantum information science, including probabilistic schemes for entangling multiple atoms in a cavity [11-13]. Although our current investigation has centered on the case of small $N \leq 3$, there are reasonable prospects for an extension to higher values $N \lesssim 10$ [see Fig. 2(c)]. Moreover, the rate at which information about $N$ is acquired can be substantially increased from the current value $\kappa|\langle\hat{a}\rangle|^{2} \sim 10^{5} / \mathrm{s}$ toward the rate $g^{2} / \kappa \geq 10^{8} / \mathrm{s} \gg \gamma$, with $\gamma$ the rate for fluorescent imaging [4].

This work is supported by the Caltech MURI Center for Quantum Networks under ARO Grant No. DAAD1900-1-0374, by the National Science Foundation, and by the Office of Naval Research.

[1] H. J. Kimble, Phys. Scr., T 76, 127 (1998).

[2] See, for example, Cavity Quantum Electrodynamics, edited by P. R. Berman (Academic, San Diego, 1994).

[3] J. McKeever et al., Science 303, 1992 (2004).

[4] C. J. Hood et al., Science 287, 1447 (2000).

[5] H. Mabuchi and A. C. Doherty, Science 298, 1372 (2002).

[6] T. Pellizzari, S. A. Gardiner, J. I. Cirac, and P. Zoller, Phys. Rev. Lett. 75, 3788 (1995).

[7] H.-J. Briegel, S. J. van Enk, J. I. Cirac and P. Zoller, in The Physics of Quantum Information, edited by D. Bouwmeester, A. Ekert, and A. Zeilinger (SpringerVerlag, Berlin, 2000), p. 192.

[8] J. Ye, D. W. Vernooy and H. J. Kimble, Phys. Rev. Lett. 83, 4987 (1999).

[9] J. McKeever et al., Phys. Rev. Lett. 90, 133602 (2003).

[10] P. Maunz et al., Nature (London) 428, 50 (2004).

[11] L. M. Duan and H. J. Kimble, Phys. Rev. Lett. 90, 253601 (2003).

[12] J. Hong and H.-W. Lee, Phys. Rev. Lett. 89, 237901 (2002).

[13] A. S. Sørensen and K. Mølmer, Phys. Rev. Lett. 90, 127903 (2003).

[14] G. R. Guthöhrlein et al., Nature (London) 414, 49 (2001); A. B. Mundt et al., Phys. Rev. Lett. 89, 103001 (2002).

[15] R. J. Cook, in Progress in Optics XXVIII, edited by E. Wolf (Elsevier, Amsterdam, 1990), p. 361.

[16] S. Kuhr et al., Science 293, 278 (2001).

[17] Cavity finesse at $\lambda_{0}$ is $\mathcal{F}=4.2 \times 10^{5}$, with each mirror reflectivity $R$ given by $1-R=7.5 \times 10^{-6}$.

[18] K. L. Corwin, S. J. M. Kuppens, D. Cho, and C. E. Wieman, Phys. Rev. Lett. 83, 1311 (1999).

[19] Peak FORT intensity is $I_{0}=3.4 \times 10^{9} \mathrm{~W} / \mathrm{m}^{2}$.

[20] H. Katori, T. Ido, and M. Kuwata-Gonokami, J. Phys. Soc. Jpn. 68, 2479 (1999); T. Ido, Y. Isoya and H. Katori, Phys. Rev. A 61, 061403 (2000).

[21] H. J. Kimble et al., in Laser Spectroscopy XIV, edited by R. Blatt, J. Eschner, D. Leibfried, and F. Schmidt-Kaler (World Scientific, Singapore, 1999), p. 80.

[22] C. J. Hood, M. S. Chapman, T.W. Lynn, and H. J. Kimble, Phys. Rev. Lett. 80, 4157 (1998).

[23] P.W. H. Pinkse, T. Fischer, P. Maunz, and G. Rempe, Nature (London) 404, 365 (2000).

[24] Theoretical studies of the $N=1$ case similar to Ref. [25] support these qualitative characteristics. Residual Zeeman splittings and the spatially dependent $\Omega_{3}$ polarization are included in the simulations.

[25] A. D. Boozer et al., Phys. Rev. A 70, 023814 (2004). 